\title{
Comunistas oficiales y extraoficiales en competencia: el rol asignado a la Internacional ante el surgimiento de la facción "chispista" en el PC de la Argentina*
}

\author{
Victor Augusto Piemonte \\ UBA/CONICET \\ augusto.piemonte@gmail.com
}

En la década de 1920 el Partido Comunista de la Argentina (PC) atravesó tres grandes crisis que acabaron confluyendo en sendos procesos de escisión: la que implicó a los "frentistas" en 1922, la que protagonizaron los "chispistas" en 1924 y aquella otra que tuvo a José Penelón en el centro de la discordia en $1927 .{ }^{1}$ En este artículo se recuperan críticamente los motivos que originaron y el modo en que se desarrolló la segunda de estas luchas faccionalistas, enmarcada dentro del proceso de bolchevización emprendido por entonces por el partido. En la resolución de este conflicto intrapartidario desempeñó un papel de primer orden la Internacional Comunista (IC), a la cual había suscripto oficialmente el PC en el III Congreso de 1921 celebrado por aquella en Moscú. Esta situación permitió reforzar toda una serie de especulaciones que durante varias décadas redundó en interpretaciones sesgadas, sostenidas principalmente por "renegados" del PC y por rivales pertenecientes a otras fuerzas de izquierda, quienes insistian en advertir la existencia de un comunismo vernáculo supeditado a la Rusia soviética desde su mismo nacimiento en enero de 1918. No obstante, en contraposición a estas interpretaciones y relativizando la naturaleza de la participación soviética, consideramos aquí que el ingreso cada vez más activo del Comité Ejecutivo (CE) de la IC en la toma de decisiones locales fue el

\footnotetext{
* El autor agradece los comentarios y sugerencias recibidos por parte de los evaluadores anónimos del presente artículo.

1. Tal como lo ha notado oportunamente H. Camarero (2013: 139), en uno de los escasos artículos de investigación referidos de manera directa a la relación entre los comunistas argentinos y los comunistas soviéticos en la década de 1930, Silvia Schenkolewsky-Kroll confunde las últimas dos grandes escisiones enumeradas al considerar que el PC "en 1928 sufrió una crisis por la cual fueron expulsados parte de sus principales activistas, los llamados "chispistas", entre los que se destaca el lider y concejal José Penelon" (1998-1999: s/p).
} 
producto de la voluntad de los comunistas argentinos y de la presión ejercida en consecuencia antes que la aplicación rigurosa en extremo de las 21 condiciones de admisión votadas por la IC en su II Congreso de 1920 (cf. Internacional Comunista, 1973: 109-114). Se habria tratado, entonces, de una "mediación" soviética más que de una "intervención" propiamente dicha. En este sentido, indagaremos acerca de la posibilidad de que haya sido el propio PC quien fue conduciendo el proceso de fagocitosis de sus propios márgenes de autonomía en favor de una relación cada vez más estrecha con Moscú que le permitiera asentar la posición de la facción mayoritaria como encarnación del comunismo oficial en el país y ganar, al mismo tiempo, mayor legitimidad entre sus partidos homólogos de la región sudamericana.

Quien previamente se ha ocupado de estudiar las primeras luchas facciosas al interior del PC con cierto detalle y mediante la consulta de importantes documentos pertenecientes a los archivos soviéticos fue el dirigente del Partido Comunista Revolucionario de la Argentina, Otto Vargas. ${ }^{2}$ Llevando a cabo una valiosa reconstrucción del debate que condujo a la ruptura cristalizada en el VII Congreso nacional de 1925, su análisis se halló orientado a tomar posición a favor del ímpetu revolucionario de la corriente llamada "ultraizquierdista" -también conocida como "chispista"-y en contra del "reformismo" de la dirección mayoritaria. Vargas observó que la compulsa entonces librada entre el comunismo "oficial" y el comunismo "extra-oficial" implicó un parteaguas para la vida interna del partido argentino. Según su perspectiva, a partir de entonces "la orientación política fundamental del Partido Comunista de la Argentina y la composición de sus órganos de dirección serian decididos por la Internacional Comunista y, en última instancia, por el Partido Comunista de la URSS" (Vargas, 1999: 271). No coincidimos aquí en el señalamiento de que es con la polémica chispista cuando el PC pierde su autonomía, sino que la misma continúa vigente hasta que se produce el alejamiento del grupo de José Penelón. Por el contrario, entendemos que, si bien la mediación soviética representó una ruptura respecto de la relación precedente entre el organismo internacional y el partido nacional, en la medida en que en adelante este último recurrirá al primero para dirimir sus conflictos, la alineación de Buenos Aires con Moscú no terminó por entonces de refinarse, hecho que finalmente tendria lugar tras la expulsión de Penelón y sus seguidores. Con este propósito, recurriremos a realizar un análisis exhaustivo de los documentos que registran la actividad entre las direcciones del PC y de la IC,

2. Una década antes que lo hiciera Vargas, Godio (1988: 167-278) había repuesto ampliamente los debates intrapartidarios del PC, aunque lo hizo poniendo el foco en la compilación documental muy suscintamente comentada antes que en el análisis crítico. 
informaciones que serán debidamente complementadas con la lectura del órgano de prensa de la sección argentina, La Internacional, en el período abordado.

\section{La Carta Abierta ante la cuestión del programa}

En sus orígenes el PC había sido antiprogramático, y había basado su política en los parámetros de una declaración poco desarrollada que instaba a la utilización de conceptos comunistas y criticaba con severidad el orden social burgués cuya destrucción promovia. Este conjunto de principios de acción había despreciado la conveniencia de defender reivindicaciones inmediatas por considerar que eran patrimonio de las corrientes reformistas. La cuestión del programa había sido discutida de manera abierta en el V Congreso partidario celebrado en julio de 1923. En aquella instancia se impuso la posición de la minoría (al que se denominará "grupo verbalista"), encabezada por Cayetano Oriolo y Teófilo González, partidaria de ratificar la declaración de principios de 1921. Aduciendo incompatibilidades entre reformas inmediatas y el "programa" vigente del partido, Oriolo y González se habian opuesto al paquete de medidas (fijación de alquileres máximos, rechazo del incremento de las tarifas tranviarias, igualdad de salarios entre hombres y mujeres) que desde la mayoría del Comité Ejecutivo del PC intentaban promover Pedro Romo y Nicolás Di Palma. ${ }^{3}$

Los planteos antiprogramáticos se basaban en la interpretación de que el partido se encontraba transitando una etapa en la cual urgía captar la atención de los dirigentes sindicales, quienes, a causa de su adscripción antipolítica, podian resultar ahuyentados si se adoptaba un programa político rígido. Las masas, por su parte, debian ser preparadas teóricamente para que pudieran comprender sin equivocos los contenidos del programa del partido cuando finalmente fuera elaborado. En definitiva, este enfoque estimaba que el programa debía reservarse para cuando el PC, habiendo cumplimentado la etapa inicial, estuviera en condiciones de dirigirse a las masas en general. Expuesta en Rusia por José Penelón y Antolín Lucendo, la declaración fue criticada por el representante del CE de la IC en Sudamérica, Alfred Stirner (seudónimo del comunista de origen suizo Edgar Woog. Cf. Jeifets, Jeifets y Huber, 2004: 340-341). Este último les anunció entonces que enviaría a la Argentina una carta en donde sería estudiada la situación del país y se evaluaria la táctica del PC según la línea política de la IC.

La Carta Abierta enviada por la IC dio paso a una serie de sesiones

3. N. Di Palma, "El viejo "programa" del partido y su pretendida justificación histórica", La Internacional (LI), año VIII, $\mathrm{N}^{\circ} 1124,2$ de septiembre de 1925, pp. 2-3. 
de debate que tuvieron lugar en el local que el PC tenía en la ciudad de Buenos Aires en la calle Estados Unidos 1056. Allí intervinieron activamente, entre otros, Francisco Loiácono, Angélica Mendoza, Teófilo González, Enrique Müller, Ghitor (Orestes Ghioldi), Antolín Lucendo, Héctor Raurich y Victorio Codovilla. ${ }^{4}$ La carta de la IC presentaba, a grandes rasgos, cinco situaciones problemáticas de porte. En primer lugar, se realizaba un estudio histórico del PC poniendo el foco en su organización, orientación y táctica, y señalaba el error en que se había incurrido al no adoptar una centralización democrática sistematizada. En segundo lugar, se efectuaba una descripción de las condiciones específicas de la Argentina. Como tercer problema, se llamaba a fijar posiciones claras en el terreno sindical, considerando que constituía esta la principal cuestión para la clase obrera. Un cuarto aspecto problemático era la lucha contra el imperialismo. La quinta y última cuestión pasaba por el problema táctico del Frente Único. ${ }^{5}$ La Carta Abierta de la IC llegó al país en abril de 1925. Fue inmediatamente publicada en La Internacional y alrededor de su contenido giraron las discusiones que en los meses siguientes ocuparon a la dirección comunista, hasta que se produjo la eyección del grupo minoritario.

Como miembro del grupo que rechazaba las reivindicaciones inmediatas, Teófilo Arfuch presentó la cuestión del programa como si fuera una más entre todos los puntos planteados por la IC para el caso de la sección argentina. No obstante, ésta era la cuestión central. Recogiendo la importancia destinada por el CE de la IC a la redacción de un programa en reemplazo de la antigua declaración, Lucendo afirmaba que esta tarea era la única que podía evitar que el partido cayera en el sectarismo $\mathrm{y}$, contrariamente, permitiria dirigir su rumbo hacia las antípodas al permitirle transformarse en un partido de masas.

Por su parte, Victorio Codovilla no dudaba en interpretar de la manera menos autónoma y más disciplinada los mensajes de la Comintern para la bolchevización de los partidos comunistas, que había sido aceptada por el PC en su III Congreso: "La I.C. en sus tesis sobre bolchevización de los partidos, insiste en cada párrafo sobre la necesidad de homogeneizar ideológicamente a los mismos, base fundamental para poder hacer de ellos partidos monolíticos". ${ }^{6}$ Por lo tanto, sin que Codovilla lo pusiera por escrito, se entendía que en su concepción la existencia de

4. "El viernes seguirá el comentario de la Carta Abierta", $L I$, año VIII, $\mathrm{N}^{\circ} 1124,2$ de septiembre de 1925, p. 2.

5. T. Arfuch, "Función de la Carta Abierta", LI, año VIII, N 1137, 17 de septiembre de 1925 , p. 2.

6. V. Codovilla, “¿Partido político o conglomerado de fracciones?”, LI, año VIII, $\mathrm{N}^{\circ}$ 1140, 20 de septiembre de 1925, p. 2. 
una disidencia al interior de la dirección del PC no haría más que obstaculizar el buen desarrollo del proceso de bolchevización e implicaba, implícitamente, un rechazo a los mensajes de aquella entidad superior que era la IC. Incluso consignaba Codovilla su sorpresa ante el hecho de que la Carta Abierta hubiera causado más desinterés que conmoción. Pese a su carácter de documento histórico fundamental, Lucendo notaba, al igual que Codovilla, que la carta habia pasado inadvertida hasta que se produjo la reunión del Comité ampliado que reflotó su importancia en tanto guía para la organización del PC.

La IC celebraba la separación dentro del PC de los elementos reformistas que, amparándose en la aplicación práctica del "frente único", emprendian una campaña contra la "dirección honrada" en un intento por disgregar las fuerzas comunistas. La existencia de una organización disciplinada y homogénea aparecía como una característica sine qua non para realizar un verdadero trabajo activo entre los obreros. Por eso sostenía la IC que el momento crítico por el que había pasado el PC a partir del desafio contrarrevolucionario planteado por los verbalistas constituía una buena ocasión para fortalecer la disciplina interna y avanzar en la bolchevización del partido. ${ }^{7}$ En efecto, desde que tuvo lugar una reunión del Comité Ejecutivo del PC el 27 de junio de 1925 (Campione, López Cantera y Maier, 2007: 34-35), la organización en células pasó a ser concebida "como la unidad fundamental y reproductora del PC, la base de su funcionamiento y el puente de vinculación entre el partido y la clase obrera, del mismo modo que el comité lo fue para la UCR y el centro o casa del pueblo, para el PS" (Camarero, 2007: 3). Un paso definitivo hacia la bolchevización debía ser dado mediante la elaboración de un verdadero programa de partido.

En el Proyecto de Programa del PC, la comisión redactora buscó las causas de la ausencia de un programa de reformas inmediatas no dentro del propio partido sino en las masas trabajadoras. Era el "estado psicológico de las masas" que siguió al triunfo de la revolución proletaria en Rusia, con la necesidad de combatir el reformismo, lo que había determinado la interpretación fallida de una situación de explosión revolucionaria a nivel mundial. El Comité de Propaganda de la IC se había ocupado de poner en aviso a las masas de que, si bien el capitalismo experimentaba una crisis general, las perspectivas de una revolución mundial inmediata no eran realistas. Sin admitir el error en que había incurrido la propia dirección comunista, sostenía ahora que la "organización y acción de clase sólo pueden hacerse sobre la base que

7. "Lettera aperta del Comitato Esecutivo della Internazionale Comunista al Partido Comunista dell' Argentina", Ordine Nuovo, año I, n 106, 4 de septiembre de 1925 (incluido en $L I$, año VIII, $\mathrm{n}^{\circ} 1126$, p. 4). 
ha constituido siempre la fuerza dinámica de la acción revolucionaria: las necesidades inmediatas de los asalariados" (el subrayado es nuestro). ${ }^{8}$

Una vez fuera del CE del PC por renuncia voluntaria, Cayetano Oriolo intervino en el debate generado en torno de la Carta Abierta de la IC. ${ }^{9}$ La redacción de La Internacional hizo explícita la intención del $\mathrm{CE}$ de abrir públicamente dicha discusión. Tras el Primer Congreso Extraordinario, celebrado en los días 25 y 26 de diciembre de 1920, los comunistas dieron forma a un programa que incluía algunas reivindicaciones inmediatas, el cual terminó siendo aprobado más tarde por el PC. Entendía Oriolo que este "reformismo de derecha" había encontrado posibilidades concretas de expresión a partir de la negativa del CE del PC a discutir cualquier actualización del programa. Asimismo, Oriolo intentó demostrar que Rodolfo Ghioldi, si bien ahora se autoproclamaba promotor de la introducción de las reivindicaciones inmediatas al programa de acción del PC, anteriormente se había revelado como uno de sus más enérgicos detractores. Antes de que Moscú pusiera el eje en las reivindicaciones, dando paso al vuelco de Ghioldi y del resto de la "dirección oportunista", sostenía Oriolo que él mismo, como parte de la minoría en el CE que conformaba junto a Teófilo González en el congreso de 1923, había pretendido incorporarlas: "Sostenemos, en principio, que no puede haber comunistas que se opongan a las reivindicaciones inmediatas, así como no puede aceptarse que los haya que se opongan a la acción parlamentaria o a la táctica de frente único", pues, en su opinión, estaba claro que existía "una diferencia fundamental entre las reivindicaciones comunistas y las de los reformistas". Pretendia Oriolo presentarse así como un inspirador del sentido leninista auténtico, que había intentado forjar la táctica comunista del "frente único" mediante la implementación de reivindicaciones inmediatas en los contenidos programáticos del PC incluso antes de que así lo dispusiera el CE de la IC. Por ende, la conducta preclara de la minoria "verbalista" quedaba implícitamente exculpada de aquellas equivocaciones tácticas previas que habian hecho del partido una secta antes que un partido de masas.

En medio de la reyerta, Oriolo manifestó que no tenía intenciones de abandonar el partido, y advirtiendo que el favor de la IC se encontraba del lado de la mayoría de la dirección argentina, pretendió presentar la cuestión del programa como una problemática zanjada. Recurrió para ello a la fuente máxima de legitimidad posible: la Carta Abierta. Aunque

8. "Proyecto de Programa del Partido Comunista de la Argentina", LI, año VIII, $\mathrm{N}^{\circ}$ 1168, 29 de octubre de 1925, p. 4.

9. Cayetano Oroiolo, "Orientación leninista de nuestro partido. Estamos con la Carta Abierta de la Internacional Comunista - No hay crisis política”, $L I$, año VIII, $N^{\circ} 1127$, 5 de septiembre de 1925, pp. 2-3. 
la misiva llevaba la firma de Jules Humbert-Droz, miembro fundador del PC de Suiza y jefe del Secretariado Latino del CE de la IC, en realidad pareció haber sido redactada por Penelón durante su estadía en Moscú y revisada por Codovilla. ${ }^{10}$ Oriolo procuró dejar en evidencia que la IC constataba "con agrado que, finalmente, el Partido Comunista de la Argentina ha reconocido su error y se apresta a corregirlo" ${ }^{11} \mathrm{La}$ autocritica había sido ya efectuada y ahora debía surtir efecto en la modificación del programa. Quien no respetaba el mandato de Moscú, violentando las expresiones vertidas por la IC en su misiva, era el propio Ghioldi, al intentar revivir sin motivo aquel asunto superado: "No hay crisis política, compañero Ghioldi; en la cuestión del programa, el Partido, por unanimidad, está con las reivindicaciones inmediatas". ${ }^{12}$ Tampoco era cierto que Oriolo, según su propia afirmación, hubiera mostrado desconfianza hacia el CE del PC; antes bien, había realizado un reclamo para que los informes que fueran en adelante elevados a la IC se dieran a publicidad dentro del partido.

Tampoco Teófilo González, así como la gran mayoría de quienes habían defendido en el pasado la prescindencia de programa, deseaban distanciarse del PC. En su defensa, González denunció entonces una operación en la que habría estado incurriendo el CE del PC al utilizar la Carta Abierta con intenciones de monopolizar una dirección partidaria que ya hegemonizaban de hecho. ${ }^{13}$ En su opinión, no se estaba valorando el sentido pedagógico plasmado por la IC en su documento para el partido argentino, sino que se lo había convertido en un instrumento de autoridad contra la minoría para proceder a su expulsión. La autocritica intrapartidaria se convertía así en un recurso de sentencia disciplinaria que vedaba toda posibilidad de reparación.

En su calidad de miembro del CE del PC, Di Palma pretendió poner al descubierto la "maniobra" de Oriolo cuando contestó su descargo, afirmando que los verbalistas no apoyaban en absoluto la utilidad de las mejoras inmediatas de las masas trabajadoras para la lucha del PC, ya que en su ideario aquéllas eran competencia de las organizaciones

10. Kersffeld (2013: 8-9) señala como cierta esta observación, aunque Vargas (1999: 275) ya había advertido la conveniencia de referirse a este hecho como una posibilidad no comprobada de manera fehaciente. Desde las filas del PC, Arévalo (1983: 20) mencionaba a comienzos de la década de 1980 únicamente la participación de Codovilla en la redacción de la misiva.

11. Cayetano Oriolo, "Orientación leninista...", ob. cit., p. 2.

12. Ibídem.

13. Teófilo González, "Aclarando conceptos programáticos", LI, año VIII, nº 1141, 22 de septiembre de 1925 , p. 3. 
sindicales. ${ }^{14}$ Por su parte, Romo respondió a Oriolo que todos los miembros del CE tenían a su disposición las copias de todos los informes que los delegados argentinos habian presentado ante la IC. No obstante, aseveraba, ni Oriolo ni Teófilo González habian manifestado ningún interés por consultarlos. ${ }^{15}$

A su turno, el chispista Francisco Loiácono acusó en el órgano oficial del partido a Ghioldi, Codovilla y Romo de ser los verdaderos oportunistas dentro del partido. ${ }^{16}$ Entendía Loiácono que este sector buscaba hacerse con el control absoluto de la dirección cuando continuaba desacreditando a la minoría que, tras la lectura y discusión de la Carta Abierta, había decidido avalar la creación de una comisión encargada de redactar un programa de reivindicaciones inmediatas. Fue Codovilla el encargado de cerrar el debate mediante una respuesta articulada en varias partes que fueron apareciendo en números consecutivos de $L a$ Internacional. Para Codovilla no podía seguir aceptando el partido entre sus filas a personalidades de probada incapacidad a la hora de asumir equivocaciones y conducir modificaciones reales para subsanarlas. Antes bien, entendia que detrás de la aceptación de la facción antiprograma se escondía un oportunismo táctico: aunque hicieran explícita la voluntad unánime de adherir al programa de reivindicaciones inmediatas, los disidentes mantenían en la práctica su rechazo a la bolchevización. Si Oriolo y sus compañeros no se decidian a abandonar el partido era para "no perder el contacto con la gran mayoría del partido y por ende no perder la oportunidad de realizar su politica antibolchevique". ${ }^{17}$

La Carta Abierta definitivamente había acabado acalorando los ánimos dentro del PC hasta niveles probablemente insospechados por la propia IC. Antes que permitir discusiones calmadas que permitieran adoptar soluciones conciliatorias, el intercambio cada vez más exacerbado se perdió en acusaciones personales que dieron paso a la conformación definitiva de dos bloques contrapuestos en el cual la posición frente a la carta de la Comintern con la que todos decían acordar sirvió para que la facción mayoritaria en la dirección se autoproclamara defensora genuina del plan de bolchevización comunista. Dentro de esta

14. N. di Palma, "La conformación ideológica de los que aceptan la Carta Abierta", $L I$, año VIII, $\mathrm{n}^{\circ} 1127,5$ de septiembre de 1925, p. 2

15. P. Romo, "Las cosas en su lugar. Contestando a Oriolo", LI, VIII, n 1133, 12 de septiembre de 1925, p. 2.

16. P. Romo, "La carta abierta de la IC y la cuestión programática a través de la historia de nuestro partido", LI, año VIII, n 1170, 31 de octubre de 1925, p. 2.

17. V. Codovilla, “¿Partido monolítico o conglomerado de fracciones?, $L I$, año VIII, $\mathrm{n}^{\circ}$ 1144, 26 de septiembre de 1925, p. 2. 
lógica, el derrotero de la compulsa tenía que ser ratificado por la IC. Y efectivamente así se hizo.

Recientemente llegado a Buenos Aires como miembro de la Juventud Comunista Holandesa (Gilbert, 2007: 56), el alemán Jean Jolles compartía en las páginas de La Internacional su juicio valorativo acerca de los enfrentamientos entre el CE y la oposición, no sin haber reconocido primero que su conocimiento sobre el tema se limitaba a las pocas asambleas realizadas a este propósito que había logrado presenciar. ${ }^{18}$ Jolles declaraba que el grupo chispista poseía una "mentalidad trotskista" contraria al verdadero leninismo. Hacía constar, también, que la separación de esta corriente "advenediza" era una cuestión de tiempo cuya concreción debía definir el CE.

Entre los cuadros partidarios y sindicales destacados que fueron distanciados del PC en el VII Congreso reunido del 26 al 28 de diciembre de 1925 y pasaron a encabezar el nuevo Partido Comunista Obrero se encontraban Angélica Mendoza, Héctor Raurich, Cayetano Oriolo, Mateo Fossa, Teófilo González, Rafael Greco (quien ya había sido expulsado en la crisis de 1922), Alberto Astudillo, José Paniale, Romeo Gentile, Modesto Fernández, Francisco Loiácono, Teófilo Arfuch. Participaron también de esta experiencia los fundadores de la revista universitaria Insurrexit editada en los años 1920 y 1921 (cf. H. Tarcus, 2000: 38-50; 2004: 750-754), Mika Feldman y su compañero Hipólito Etchebéhère, quien acababa de regresar al país tras ser enviado a La Habana en calidad de delegado del Secretariado Sudamericano de la IC para participar, junto a Enrique Flores Magón, del congreso de refundación del PC cubano (V. Jeifets y L. Jeifets, 2009-2010: 58). También pasó a integrar sus filas Miguel Contreras, aunque lo hizo por poco tiempo ya que decidió retornar al PC.

La facción triunfante aprovechó el visto bueno de autoridad recibido para señalar a Moscú que aquello que podía haber parecido una debilidad era en realidad un signo de fortaleza. Con posterioridad a las expulsiones, Paulino González Alberdi publicó un artículo en el órgano del partido, en donde presentaba la emergencia de la posición "oportunista" como el producto de una crisis de crecimiento del PC. Esta crisis interna que, en su opinión, había podido destruir al partido en otro tiempo, ahora no hacía más que fortalecer su unidad y despejar su camino para la bolchevización y su consiguiente transformación en un partido de masas. ${ }^{19}$

18. Jean Jolles, "Trotskismo y Leninismo en el Partido Comunista de la Argentina", LI, año VIII, $\mathrm{n}^{\circ} 1149,2$ de octubre de 1925, p. 2.

19. Paulino González Alberdi, "Preparando el Congreso. El grupito opositor: una 


\section{La consolidación orgánica del chispismo: el Partido Comunista Obrero en busca del reconocimiento de Moscú}

Encarnando la minoria dentro de la dirección del PC, la corriente verbalista había hegemonizado los congresos V y VI del PC celebrados respectivamente en los meses de julio de 1923 y 1924, haciéndose con la voluntad del grueso de los afiliados. No obstante, la mayoria de la dirección del PC logró hacerse con el beneplácito de Moscú, lo que permitió forzar la expulsión de los ultraizquierdistas en vísperas del VII Congreso el 26 de diciembre de 1925. Fue por esta situación de "poder bifurcado" al interior del PC que las mediaciones cominternianas que dieron "la razón a las opiniones de las mayorías en los órganos de la dirección" no se proyectaron "con claridad en el conjunto de afiliados partidarios" (Campione, 2007: 172-173).

En una asamblea general celebrada el 16 de enero de 1926, los verbalistas expulsados del PC tomaron la resolución de formar el Partido Comunista Obrero (PCO) y editar un órgano propio, La Chispa, el cual les proporcionó su nuevo nombre de "chispistas". Desde entonces el PCO intentó erigirse en la "verdadera vanguardia" del proletariado argentino y buscó "construir un movimiento comunista distinto y alternativo" (Kersffeld, 2007: 37) al "comunismo oficial", poniendo en su programa un marcado énfasis en la cuestión del imperialismo dentro de la región latinoamericana. Vale recordar que la IC había cuestionado la subvaloración que el grueso del CC del PC efectuaba a propósito de la presencia de expresiones antiimperialistas en América Latina a partir de una coyuntura internacional de expansión de los capitales monopolistas inglés y norteamericano que redundaba en la posibilidad de una guerra $;{ }^{20}$ por lo tanto, el chispismo se presentó como una opción concreta para revertir esta falencia. Así, en contraposición a la apoplejía de la dirección triunfante en el PC, el órgano del PCO emergía "para continuar en la Argentina, modestamente, la obra revolucionaria de la Iskra en sus primeros tiempos, cuando Lenin la dirigía". ${ }^{21}$ Fueron designados para ocupar el Comité Central (CC) del nuevo partido Cayetano Oriolo (nombrado también para ocupar el puesto de secretario general), Teófilo

piedrecilla que obstaculiza el camino hacia la transformación en Partido de masas. Conclusión”, LI, año VIII, nº 1154, 8 de octubre de 1925, p. 2.

20. "Lettera aperta del Comitato Esecutivo della Internazionale Comunista al Partido Comunista dell' Argentina”, Ordine Nuovo, año I, n 104, 2 de septiembre de 1925 (incluido en $L I$, año VIII, $\mathrm{n}^{\circ} 1124$, p. 4).

21. "Creación y Organización del Partido Comunista Obrero. Causas que las originan", La Chispa, año I, n 1, 30 de enero de 1926, p. 2. 
González, Angélica Mendoza (también directora de La Chispa), Modesto Fernández, Rafael Greco, Francisco Loiácono, E. Satanoski, S. Scavelli y Mateo Fossa. ${ }^{22}$ Para la dirección del PC estaba claro que a través de $L a$ Chispa, los disidentes que se habían congregado en el PCO se revelaban como "perfectos instrumentos policiales". ${ }^{23} \mathrm{El} \mathrm{CE}$ del PC adjuntaba en un envío a Moscú un ejemplar del periódico para que en la IC sacaran sus propias conclusiones. El 26 de mayo de 1926 el CC de la IC envió a Buenos Aires una carta firmada por su secretario Jules Humbert-Droz solidarizándose con las tareas de bolchevización llevadas a cabo por la dirección mayoritaria y criticando a los chispistas por su intención de sabotear el trabajo clasista del PC. ${ }^{24}$

De igual modo, la IC resolvió enviar al Secretariado Sudamericano, cuyas funciones inmediatas consistian en regularizar los contactos entre Moscú y los partidos comunistas sudamericanos, una nueva misiva que llevaba la firma de Ercoli (seudónimo del líder del PC italiano Palmiro Togliatti) e incluía un pedido para que fuera publicada en los órganos comunistas y afines al comunismo de América. En ella se denunciaba el carácter anticomunista del grupo chispista, el cual continuaba lanzando panfletos en los que repudiaba la acción de los dirigentes del PC. La carta constituía un intento por revertir los dichos volcados en La Chispa y descalificar, al mismo tiempo, al grupo que se organizaba en torno a sus páginas.

Las pretendidas declaraciones de amistad hacia la Rusia de los Soviets y el Comintern, hechas por ese grupo, no son más que la máscara que sirve para velar su propósito contrarrevolucionario. No se puede ser amigo de la revolución soviética y de la Comintern cuando se combate por todos los medios - por el asesinato inclusive- los Partidos Comunistas que constituyen la base segura no solamente de la primera Revolución Proletaria, sino también un punto de apoyo para el desarrollo de la revolución mundial. La Comintern conoce ya esta táctica que ha sido empleada por todos los traidores a la causa comunista: simular, en las palabras, ser amigo del movimiento comunista internacional, pero de hecho buscar a disgregar los Partidos Comunistas de sus países.

22. "Partido Comunista Obrero", La Chispa, año I, no 1, 30 de enero de 1926, p. 4.

23. Partido Comunista de la Argentina, Sección de la I.C., al compañero HumbertDroz, secretario de la Comintern, Buenos Aires, 4 de febrero de 1926, Archivo de la Internacional Comunista, Biblioteca del Congreso de la Nación Argentina [en adelante Archivo IC, BCNA], rollo [r.] 1, sección [s.] 2, p. 3.

24. "La Internacional Comunista denuncia a una agrupación enemiga del Comunismo", La Correspondencia Sudamericana, año I, nº 5, 15 de junio de 1926, pp. 1-2. 
En consecuencia, nosotros ponemos en guardia a las organizaciones revolucionarias de América contra esos pretendidos "comunistas obreros" y las invitamos a denunciar, como ya lo ha hecho la Comintern, a esos elementos como enemigos del comunismo y como agentes patronales. ${ }^{25}$

Esto equivalía a otorgar, una vez más, el apoyo del comunismo soviético a la conducción comunista argentina. Era el PC, y no su desprendimiento en el PCO, la auténtica encarnación de la vanguardia de la clase obrera argentina. Estas críticas iniciales no impidieron a los comunistas disidentes reunidos en el PCO intentar el reestablecimiento de vínculos con Moscú. En efecto, sus dirigentes no se privaron de enviar ellos mismos un informe a la IC. Comunicaban allí que el I Congreso del PCO, reunido en agosto de 1927, había decidido que se contactaría a la IC con el fin de solicitar que ésta estudiara los problemas que conllevaba la coexistencia de dos partidos comunistas en la Argentina. Lógicamente, el PCO se presentaba a sí mismo como el auténtico partido comunista en el país, en tanto que el PC había resultado víctima de las malas artes de una "dirección oportunista". En el informe se aseguraba que, si bien formalmente el PCO se encontraba fuera de la IC, en la realidad se había adaptado a las líneas políticas emanadas por el organismo comunista internacional. En su congreso inaugural el PCO resolvió por unanimidad ratificar "su adhesión incondicional a la Internacional Comunista". ${ }^{26}$

Lejos de sentirse identificados con la representación peyorativa que de ellos había formulado la dirección mayoritaria del PC cuando afirmaba que se trataba de una "media docena de enemigos de la bolchevización", los miembros del PCO aseguraban ser los "fieles soldados" 27 de la IC. La participación chispista en la dirección de la Unión Obrera Local dentro de la Unión Sindical Argentina y la edición relativamente exitosa de su órgano La Chispa (6.000 ejemplares distribuidos en la Capital y el interior del pais) eran destacados por el PCO para refrendar sus palabras. Incluso antes de que lo hiciera el partido comunista oficial desde La Internacional, los chispistas fueron verdaderos pioneros a la hora de utilizar la prensa para criticar a Trotsky y ensalzar así todavía más la figura de Stalin (Kersffeld, 2013: 10).

25. "Declaración del Secretariado del Comintern a todos los Partidos Comunistas y organizaciones revolucionarias de América", La Correspondencia Sudamericana, año I, $\mathrm{n}^{\circ} 16,30$ de noviembre de 1926, pp. 1-2

26. "El Partido Comunista Obrero realizó el primer Congreso Comunista del país", La Chispa, año II, $\mathrm{n}^{\circ}$ 40, 27 de agosto de 1927, p. 1.

27. Informe del Comité Central del Partido Comunista Obrero de la República Argentina al Comité Central de la Internacional Comunista, septiembre de 1927, Archivo IC, BNCA, r. 5, s. 37 [En francés]. 
Para los chispistas había sido el CE triunfante en el VII Congreso el que había malogrado la relación con la IC en favor de la obtención de réditos personales. Dentro del PCO notaban cómo esta intención de acumular poder dentro del partido decantaba en el ejercicio de una "propaganda exclusivamente internacional". Es decir, una vez producido el alejamiento del sector opositor al CE, continuaron las maniobras para tergiversar el sentido de la conformación del PC en sección argentina de la IC, la cual

[...] no ha sido en ningún instante la vanguardia del proletariado nacional, sino simplemente el registrador de la conciencia confusa y de los intereses aún indefinidos de la clase obrera. La prueba más acabada de esa afirmación, es la ausencia de estrategia en la orientación en la política del Partido y de la clase obrera. ${ }^{28}$

El reclamo de reconocimiento por parte de la IC que llevaba a cabo el PCO cobró posteriormente mayor intensidad cuando en el partido ingresaron aquellos que habian abandonado el PC en su última ruptura de 1928, producida por la facción que era conducida por el hasta ese momento jefe del Secretariado Sudamericano de la IC. Desde entonces, se señalará desde el PCO que es el propio Penelón quien "denuncia a la dirección [mayoritaria del PC] de ser agentes del capitalismo infiltrados en el movimiento comunista y de agentes policiales". ${ }^{29} \mathrm{El}$ elevado número de escisiones significativas experimentadas con elevada frecuencia por el PC -la primera en 1922, la segunda en 1925 y la tercera en 1927-1928-, la participación en la escena política argentina de tres partidos comunistas diferentes (ahora que se sumaba también el Partido Comunista de la Región Argentina de Penelón), afectaban de manera inevitable el prestigio del comunismo entre los obreros, favoreciendo así a sus competidores socialistas. En la perspectiva chispista, esto no implicaba una unificación de criterios entre el grupo de Penelón y el comunismo obrero. Antes bien, los comunistas obreros intentarian aprovechar en su favor los conflictos recientes entre las distintas facciones para mostrarse ante la IC como la única fuerza comunista homogénea y unificada. En el PCO no olvidaban que en la compulsa que les costó su expulsión del PC, Penelón había defendido a Codovilla ante las acusaciones por malversación de fondos realizadas por Oriolo (cf. Oriolo, 1994). Bajo la óptica chispista, el penelonismo, al igual que

28. "Creación y organización del Partido Comunista Obrero. Causas que las originan", La Chispa, año I, $\mathrm{n}^{\circ}$ 1, 30 de enero de 1926, p. 1.

29. Carta del Partido Comunista Obrero al secretario de la Internacional Comunista, 28 de enero de 1928, p. 1, Archivo IC, BNCA, r. 5, s. 37. 
el "comunismo oficial", tergiversaba los hechos según su propia conveniencia. Aunque contara con las menores posibilidades de hacerse con el beneplácito de la IC, habiendo sido ya duramente criticado en Moscú, el PCO iba a potenciar la ruptura subsiguiente para mostrarse como la única opción sólida posible tanto frente al desbaratado PC -o "ex P.C.", como pasó a ser denominado por la prensa chispista-, ${ }^{30}$ como frente al inestable Partido Comunista de la Región Argentina de Penelón. De lo contrario no se explica por qué los chispistas eligieron este momento en particular para entablar conversaciones con el movimiento comunista internacional radicado en Moscú.

Sin embargo, dos semanas más tarde, el PCO hizo una propuesta de unidad al PC y al Partido Comunista de la Región Argentina y se apresuró a enviar una copia de su proposición escrita a la IC. ${ }^{31}$ Llegado el caso de que se produjera un muy probable rechazo a esta repentina invitación por parte de los dos nucleamientos interpelados, por entonces lejos de cualquier intento certero de pacificación, podrían argüir que habian hecho todo cuanto les era posible para evitar que continuara el faccionalismo que obstaculizaba el trabajo en profundidad con las masas trabajadoras. Y de hecho el propio PCO dejaba en claro lo titánico de su empresa al señalar que en las condiciones vigentes "los tres partidos en que se encuentra dividido el movimiento comunista en el país poseen posiciones ideológicas y tácticas completamente antagónicas que determinan una lucha previa a toda acción efectiva". ${ }^{32}$ Como parte crucial de esta misma operación, el PCO llamaba a la IC a colaborar en la solución de la crisis que experimentaba su sección argentina. Bien puede ser que, como afirmaban sus mismos promotores, el PCO no actuara motivado en un afán por expandir sus áreas de influencia. A fin de cuentas, incluso en la IC reconocían que, mientras se demoraba el PC en definir una política sindical a nivel nacional, los chispistas continuaban ganando posiciones en el movimiento obrero. Pero sin dudas la proposición se hallaba atravesada por el interés en lograr la aceptación del grupo por parte de la IC. ${ }^{33}$ Los chispistas siguieron manifestándose partidarios de la unidad comunista aun cuando la propuesta del comunismo obrero no encontró los resultados esperados. ${ }^{34}$

30. "Permanente", La Chispa, año I, n 2, 13 de febrero de 1926, p. 1.

31. Carta al Secretario de la Comisión de Control de la IC [firmada por Loiácono, secretario general del PCO], 17 de febrero de 1928, Archivo IC, BNCA, r. 5, s. 37.

32. Cf. "Frente a la nueva escisión, el partido Comunista Obrero propone la unificación de las fuerzas comunistas", La Chispa, n 52, III, 11 de febrero de 1928, p. 1.

33. Ibídem.

34. "El partido Comunista Obrero mantiene su posición unionista", La Chispa, año III, $\mathrm{n}^{\circ} 53,25$ de febrero de 1928, p. 1 
Es interesante destacar que, a pesar de que buscaba denodadamente el apoyo de la IC, la conducción del PCO no dudó en recriminarle a aquella su falta de compromiso a la hora de combatir a la "facción oportunista". El desarrollo del PC había involucionado en lugar de avanzar en cierta medida por la irresponsabilidad de la IC, que al aceptar sin reservas la validez de los informes tendenciosos que le eran suministrados, no había hecho nada para deponer a la dirección mayoritaria. Pero en lugar de realizar un análisis serio de la situación argentina, la IC había optado en todo momento por desentenderse del problema con premura, y el método para hacerlo había consistido en fallar siempre a favor de la mayoría. Pero ahora, según deducía de los argumentos expuestos, la IC se enfrentaba a la posibilidad de revertir el facilismo y de obrar con justicia en interés del movimiento comunista. Una vez más, era la IC mediante "una amplia intervención" la única que podía "solucionar la crisis permanente de la sección [argentina] de la IC". ${ }^{35}$

El Secretariado de la IC dio entonces a conocer sus fuertes criticas contra el PCO. En opinión del organismo comunista internacional, el chispismo había llevado adelante una campaña de difamación contra el comunismo, tanto por medio de La Chispa como a través de la impresión de panfletos y circulares. ${ }^{36}$ La IC se expedia sobre estas actividades de "carácter anti-comunista" en base a los malestares que los comunistas argentinos le remitian. Ya había dado su apoyo al CE del PC cuando se produjo el envío de la célebre Carta Abierta apoyando lo que entendía era una lucha justificada contra aquellos que intentaban sabotear la organización revolucionaria de los trabajadores argentinos. La misiva enviada por los chispistas a la IC solicitando su incorporación al partido mundial del proletariado no eran, para el Secretariado, más que una farsa para disfrazar su intencionalidad contrarrevolucionaria. Como parte de la situación de crisis interna experimentada por el CE del PC ante la consolidación de la facción chispista, la IC decidió exigir a su sección argentina que cesara de inmediato toda controversia pública. ${ }^{37}$

Por su parte, Próspero Malvestiti, representante sindical de Buenos Aires y delegado del PC en el IV Congreso de la Profintern de 1928, destacó el hecho de que la existencia de facciones al interior del PC era incongruente con su existencia como sección de la IC, ya que implicaba un fraccionamiento de la influencia comunistas entre las masas tra-

35. Carta del Partido Comunista Obrero al secretario de la Internacional Comunista, 28 de enero de 1928, p. 4, Archivo IC, BNCA, r. 5, s. 37.

36. Declaraciones del Secretariado de la IC a los Partidos Comunistas y organizaciones revolucionarias de América, 1928, Archivo IC, BNCA, r. 1, s. 13 [En francés]. 37. 15 de marzo de 1928, Archivo IC, BNCA, r. 1, s. 13 [En francés]. 
bajadoras, en las cuales reinaba como consecuencia el desconcierto. ${ }^{38}$ La única beneficiaria ante esta disgregación de fuerzas revolucionarias era la burguesía. Como forma adecuada para revertir esta situación, Malvestiti proponía que se celebrara de urgencia un Congreso Nacional en el que debian confluir los miembros de los tres Partidos Comunistas de Argentina: el PC y sus dos desprendimientos. La IC debía encargarse de supervisar la unificación de las partes concurrentes.

Sin embargo, el CE de la IC entendía que a la hora de discutir y resolver aquellos problemas que hacian al desarrollo de su actividad política cotidiana, el PC adoptaba formas organizativas patriarcales que no se hallaban en consonancia con las tareas que requeria llevar a cabo el partido para avanzar en el incremento de su influencia entre los trabajadores. No existía por parte del CC del PC un verdadero trabajo de dirección colectiva, sino que se hacía pesar el personalismo de algunos miembros destacados de la dirección. Esta situación relegaba al partido a un lugar de mero instrumento propagandista, carente de incidencia real entre los obreros argentinos. Era justamente la contradicción existente entre "los métodos de trabajo y de organización y las tareas del Partido" lo que contribuía "a que la discusión política degenere en lucha fraccional". ${ }^{39}$

En un informe para la IC elaborado por Codovilla, el futuro líder del PC hablaba sobre las tareas inmediatas de Penelón, el líder contemporáneo del comunismo argentino, con el resto de los partidos sudamericanos, y dejaba en evidencia las aspiraciones que debian motivar la mediación argentina cuando afirmaba la necesidad imperativa de que, haciendo uso de su investidura como integrante del CE de la IC, "se traslade cuanto antes a esos países [sudamericanos], constate de visu las deficiencias, para poder luego mantener una estrecha relación con esos P. Comunistas, y someterlos sobre las directivas del Comintern". ${ }^{40}$ Es decir, en estos años la actitud del PC no era ni de autonomía ni de subordinación a la IC. Se manifestaban todavía espacios para la convivencia de la discrepancia contraria al mecanicismo dogmático y la aceptación sin objeciones. Antes de la expulsión de los penelonistas, concretada en 1928, el perfil de la dirección argentina se encontraba mucho menos definido de lo que habria de estarlo a partir de entonces.

38. Carta de Próspero Malvestiti dirigida al Secretario de la Comisión argentina, 12 de febrero de 1928, Archivo IC, BNCA, r. 1, s. 13. Cf. también Carta de Prospero Malvestiti a Humbert Droz, Moscú, 14 de abril de 1928, Archivo IC, BNCA, r. 1, s. 13 [En francés].

39. Carta del Presidium de la IC sobre resolución argentina, 9 de abril de 1928, p. 1, Archivo IC, BNCA, r. 1, s. 9. [En francés, traducción propia]

40. Resumen sobre la situación política, económica y sindical de la Argentina para el CE de la IC, firmado por Codovilla, 1924, p. 11, Archivo IC, BNCA, r. 3, s. 22. 
Hasta ese momento, toda una suerte de contradicciones tuvieron lugar en su seno, habilitando el surgimiento de luchas intestinas, entre las cuales aquella encarnada por el chispismo fue una de las que mayores repercusiones iba a suscitar en el seno del partido.

\section{Consideraciones finales}

En la versión oficial presentada por el CE del PC, era la oposición a la consigna de "ir hacia las masas", propia de la línea de la bolchevización del partido, lo que provocaba una nueva escisión en 1925. Los chispistas fueron acusados de sabotear el contenido del programa votado en el VI Congreso del PC, celebrado del 25 al 27 de julio de 1924, para promover, en su reemplazo, toda una suerte de "reivindicaciones radicalizadas que escaparian a la comprensión de las masas obreras, sobre todo en un país como éste en que carecen de la más elemental educación política" ${ }^{41} \mathrm{La}$ Carta Abierta enviada por la IC constituía una crítica severa a la "corriente extremista-verbalista", al señalar que el PC, integrado a una realidad nacional ajena a graves desbarajustes económicos, sociales y políticos, debía dar cuenta de las reivindicaciones concretas e inmediatas a la hora de actualizar su programa de lucha (Comisión del Comité Central del Partido Comunista, 1947: 55). Aunque en un primer momento la minoría del CC rechazó la carta de la IC, muy pronto pasó a considerar la necesidad de adaptarse a su contenido en la intención de no destruir el principal factor de legitimidad con que podía contar. $Y$ es que dentro del partido podían, en estos años, tener lugar expresiones de descontento hacia algunas imposiciones de la Comintern, pero el espacio de maniobrabilidad para conducirse en ese sentido tenía límites precisos. El episodio que dio lugar al desprendimiento chispista permite advertir de manera inmejorable que la cuestión de la autonomía relativa respecto del organismo internacional con base en Moscú quedaba severamente limitada cuando se debía dar satisfacción a una compulsa interna. El segmento mayoritario recurría en tales circunstancias a obtener el reconocimiento de la máxima autoridad del comunismo mundial, y la minoría no encontraba otra respuesta inmediata más que suscribir ella misma a las soluciones propuestas por la IC.

Ni la lógica de acudir a la IC para la resolución de los conflictos internos fue una novedad en 1924-1925 (hecho que habia tenido lugar también al producirse la escisión frentista), ni fue tampoco allí cuando el partido perdió su autonomía en favor de aplicaciones más mecánicas de las fórmulas políticas soviéticas. En una respuesta brindada por el

41. Carta de Pedro Romo al CE de la IC, 4 de noviembre de 1925, p. 2, Archivo IC, BNCA, r. 3, s. 19. 
Presidium de la Internacional Comunista a los informes y documentos enviados por el PC sobre su situación a mediados de 1926 se tocaban varios puntos fundamentales, entre los cuales se dejaba constancia de la correcta interpretación que había efectuado el CC del PC sobre la Carta Abierta elaborada por la IC en enero de $1925 .{ }^{42}$ La conducción mayoritaria se había revelado, a los ojos de la IC, como un conjunto de comunistas honestos cuya capacidad y disciplina quedaban expuestas en los buenos resultados obtenidos en la campaña de bolchevización emprendida en la Argentina. Los primeros pasos para el desarrollo del $\mathrm{PC}$ en un partido de masas estaban dados. Ante el surgimiento de la facción intrapartidaria "verbalista" contraria a la acción favorable a las reivindicaciones inmediatas, la cual habiendo ganado la mayoria de los votos de los afiliados en los congresos de 1923 y 1924 constituia un lastre del cual debía desembarazarse el PC, el CE de la IC dio abiertamente su apoyo al sector mayoritario de la dirección. Así, el conflicto interno finalmente se veía resuelto con la expulsión de los "verbalistas".

Hemos visto que en el recorte temporal aquí realizado la dirección del partido se conservó en un puñado de manos que retuvo el control de los puestos esenciales para la toma de decisiones. Es en este recorrido que nuestro estudio permite concluir la presencia dentro de la vida del PC de una realidad compleja y cambiante, en la cual se advierten momentos divisorios centrales de su historia más joven: una primera etapa en donde se generan resquicios dentro de la dirección para la emergencia de una facción que desafía a la mayoría del Comité Central y plantea formas de participación política que encuentran apoyo entre los afiliados de base, y una segunda etapa en la que la mayoria de la dirección se abroquela forzando la anulación de la facción novel y acudiendo de manera cada vez más notoria a la intervención del CE de la IC para la resolución de sus conflictos internos. En otras palabras, ni el PC nació atado a Moscú, ni fueron los comunistas soviéticos los que empujaron al PC a convertirse en su discípulo más atento de Sudamérica.

Queda pendiente el desarrollo de aquella otra línea de investigación central que se desprende de este estudio y que consiste en analizar el avance en la reducción de la autonomía de la dirección del PC respecto de la dirección de la IC a partir de la expulsión del grupo de Penelón. Este episodio clave permitirá arrojar nueva luz acerca de la alineación cada vez más estrecha de los líderes del PC con los planteos emergidos en Moscú, experiencia que, una vez más urge recordarlo, formó parte de un proceso y no fue un producto congénito ni prematuro del surgimiento del comunismo en la Argentina.

42. Carta del Presidium de la IC al CC del PC, 12 de mayo de 1926, Archivo IC, BNCA, r. 1, s. 9 [En francés]. 


\section{Bibliografia}

Arévalo, Oscar (1983), El Partido Comunista, Buenos Aires: CEAL.

Camarero, Hernán (2007), A la conquista de la clase obrera. Los comunistas y el mundo del trabajo en la Argentina, 1920-1935, Buenos Aires: Siglo Veintiuno.

Camarero, Hernán (2013), "Antiguas controversias, nuevos enfoques: clase obrera, sindicalismo y comunismo en la Argentina durante la primera mitad del siglo XX. Un estado de la cuestión", PolHis. Boletín Bibliográfico Electrónico del Programa Buenos Aires de Historia Política, año 6, $\mathrm{N}^{\circ} 11$, pp. 129-147.

Campione, Daniel (2007), "El Partido Comunista de la Argentina. Apuntes sobre su trayectoria", en Elvira Concheiro, Massimo Modonesi y Horacio Crespo (coords.), El comunismo: otras miradas desde América Latina, México DF: Universidad Nacional Autónoma de México, pp. 167-215.

Campione, Daniel, Mercedes López Cantera y Bárbara Maier (2007), Buenos Aires-Moscú-Buenos Aires. Los comunistas argentinos y la Tercera Internacional. Primera parte (1921-1926), Buenos Aires: Ediciones del CCC.

Comisión del Comité Central del Partido Comunista (1947), Esbozo de Historia del Partido Comunista de la Argentina. (Origen y desarrollo del Partido Comunista y del movimiento obrero y popular argentino), Buenos Aires: Anteo.

Gilbert, Isidoro (2007), El oro de Moscú. Historia secreta de la diplomacia, el comercio y la inteligencia soviética en la Argentina, Buenos Aires: Sudamericana (2da. edición).

Godio, Julio (1988), El movimiento obrero argentino (1910-1930). Socialismo, sindicalismo y comunismo, Buenos Aires: Legasa.

Internacional Comunista (1973), Los cuatro primeros congresos de la Internacional Comunista. Primera parte, Córdoba: Cuadernos de Pasado y Presente.

Jeifets, Víctor y Lazar Jeifets (2009-2010), "Comunismo en Cuba y México. Parte I", Memoria. Revista de politica y cultura, N²39, pp. 54-59.

Jeifets, Victor, Lazar Jeifets y Peter Huber (2004), La Internacional Comunista y América Latina, 1919-1943. Diccionario biográfico, Moscú-Ginebra: Instituto de Latinoamérica de la Academia de Ciencias-Institut pour l'historie du communisme.

Kersffeld, Daniel (2007), "La prensa politica y las revistas de los comunismos latinoamericanos y sus disidencias", ponencia presentada en las $I V$ Jornadas de Historia de las Izquierdas, CeDInCI, pp. 37-52 [En Internet: http://www.cedinci.org/jornadas/4/M4.pdf].

- (2013), " "Chispismo" y comunismo: crónica de una disidencia en la izquierda argentina de los años 20”, Revista Estudios, vol. 26, N 1 , San José de Costa Rica, pp. 1-23.

Oriolo, Jordán (1994), Antiesbozo de la historia del Partido Comunista (19181918), 2 vols., Buenos Aires: CEAL. 
Schenkolewski-Kroll, Silvia (1998-1999), "El Partido Comunista en la Argentina ante Moscú: deberes y realidades, 1930-1941", Estudios Interdisciplinarios de América Latina y el Caribe, vol. 10:2, s/p.

Tarcus, Horacio (2000), "Historia de una pasión revolucionaria. Hipólito Etchebehere y Mika Felman, de la reforma universitaria a la guerra civil española", El Rodaballo, año VI, N 11/12, pp. 38-50.

- (2004), "Revistas, intelectuales y formaciones culturales izquierdistas en la Argentina de los veinte", Revista Iberoamericana, vol. LXX, $\mathrm{N}^{\circ} 208$ 209, pp. 749-772.

Vargas, Otto (1999), El marxismo y la revolución argentina, t. II, Buenos Aires: Agora.

$$
* * *
$$

Resumen: En 1925 tuvo lugar la segunda escisión del Partido Comunista de la Argentina (PC), originada en el surgimiento de la facción denominada "chispista". Durante este proceso de ruptura la Internacional Comunista (IC) desempeñó un rol de primer orden. Nuestra hipótesis es que la manera en que tanto la facción "chispista" como la mayoría de la dirección del PC decidieron resolver el conflicto, acudiendo por iniciativa propia a la intermediación de la IC, permite dar cuenta de la existencia de una intervención solicitada por la sección argentina y no impuesta por Moscú. Esta hipótesis es puesta a prueba mediante el trabajo de archivo de procedencia soviética y con la prensa comunista local. La búsqueda del reconocimiento de la IC por parte de los comunismos "oficial" y "extraoficial" fue continuada incluso después de producida la ruptura "chispista" a partir de la acción del flamante Partido Comunista Obrero.

Palabras clave: Partido Comunista - Internacional Comunista - facción chispista - bolchevización

\begin{abstract}
The second breakaway of the Communist Party of Argentina (PC) in 1925 was originated by the emergence of the "chispista" faction. The Communist International had a role of paramount importance during this breakaway process. I propose that the way in which the "chispista" faction as well as the majority of the leaders of the PC decided to solve the conflict by resorting to the Commintern's mediation, enables us to account for the existence of an intervention requested by the Argentine section and not imposed by Moscow. I put this hypothesis to test through research on the Soviet archive and the communist Argentine press. Both the "official" and the "extra-official" communists sought the acknowledgement by the Commintern even after the breakaway of the "chispista" faction through the action of the brand new Communist Workers' Party.
\end{abstract}

Key words: Communist Party - Communist International - "chispista" faction - bolshevization

Recepción: 25 de febrero de 2014. Aprobación: 30 de abril de 2014 . 